\title{
Renal Denervation: Back to the Future?
}

\author{
Venkatesh K Raman ${ }^{1}$, Paola Chrysostomou ${ }^{2}$ and Vasilios Papademetriou ${ }^{2 *}$ \\ ${ }^{1}$ MedStar Heart \& Vascular Institute, MedStar Georgetown University Hospital, Washington DC, USA \\ ${ }^{2}$ Veterans Affairs Medical Center and Georgetown University, Washington DC, USA
}

"Corresponding author: Vasilios Papademetriou, VA Medical Center, 50 Irving Street, NW, Washington DC, 20422, USA, Tel: 202-745-8334; Fax: 202-745-8636; Email: vpapademetriou@va.gov

Rec date: Apr 05, 2016; Acc date: May 13, 2016; Pub date: May 20, 2016

Copyright: ๑ 2016 Raman VK, et al. This is an open-access article distributed under the terms of the Creative Commons Attribution License, which permits unrestricted use, distribution, and reproduction in any medium, provided the original author and source are credited.

\begin{abstract}
Hypertension is a global health epidemic, affecting up to one-third of adults. Despite a plethora of pharmacologic agents that lower blood pressure and mitigate end-organ damage as well as cardiovascular events, a significant percentage of patients fail to achieve target reduction. A subset of these patients is considered to have resistant hypertension with markedly elevated blood pressure despite treatment using at least three agents, including a diuretic. With significant excess risk, additional treatment options for these patients remain an unmet need.
\end{abstract}

Keywords: Hypertension; Sympathectomy; Perinephritis; Hematuria

\section{Introduction}

Hypertension is a global health epidemic, affecting up to one-third of adults [1]. Despite a plethora of pharmacologic agents that lower blood pressure and mitigate end-organ damage as well as cardiovascular events, a significant percentage of patients fail to achieve target reduction [2]. A subset of these patients is considered to have resistant hypertension with markedly elevated blood pressure despite treatment using at least three agents, including a diuretic. With significant excess risk, additional treatment options for these patients remain an unmet need.

The sympathetic nervous system (SNS) plays an important role in the development and maintenance of hypertension, and a pivotal role in the development of drug-resistant hypertension (Figure 1). Increased sympathetic outflow is transferred from the brain to the periphery, though the sympathetic fibers and can increase heart rate and left ventricular contractility, can affect peripheral vasculature and peripheral resistance and can also affect metabolic activity in other organs. Signals are transferred to the kidney through the efferent sympathetic fibers and can stimulate the production of plasma renin, can promote absorption of sodium and water and can enhance the production of renal norepinephrine (NEPI). On the other hand afferent sympathetic fibers can transfer signals from the kidney to the brain (particularly in the presence of even minor kidney injury) and can increase sympathetic outflow. Thus the current thinking is that signal trafficking through both, efferent and afferent sympathetic fibers, that course in the adventitia of the renal artery contributes the development and maintenance of resistant hypertension. Mechanical interruption of the SNS to treat patients with malignant hypertension was implemented via surgical transection almost one hundred years ago, prior to the availability of anti-hypertensive medications, and included the spectrum of thoracolumbar sympathectomy to renal nerve resection. These were ultimately abandoned due to significant mortality, morbidity and side effects with profound orthostatic hypotension following sympathectomy. The advent of effective pharmacologic therapy for the treatment of hypertension in the late 50s renders surgical sympathectomy unnecessary for the majority of patients.

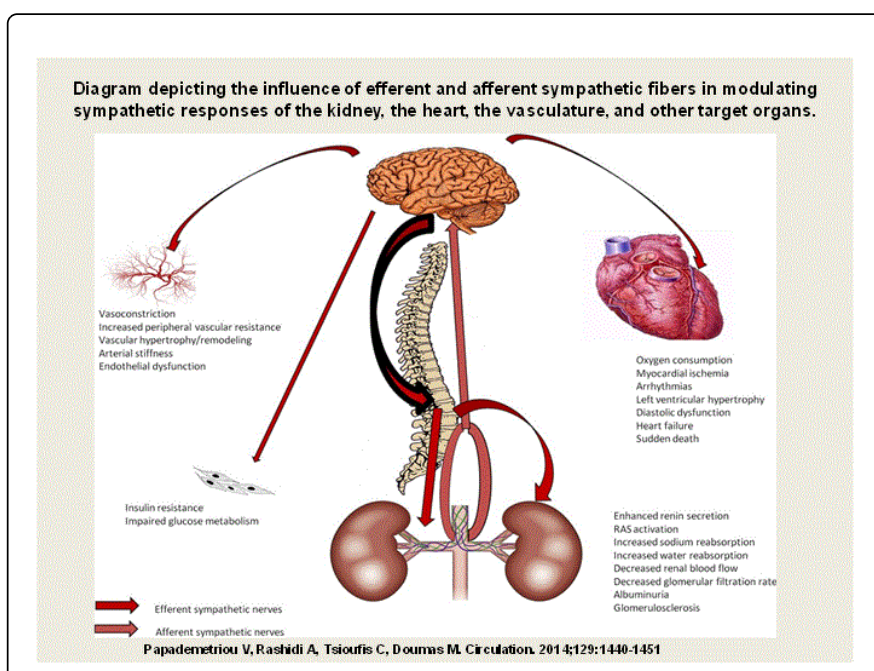

Figure 1: Influence of efferent and afferent sympathetic fibers in modulating sympathetic responses of the kidney, the heart, the vasculature and other target organs.

Developments in trans catheter technologies allowed the resurrection of this strategy to target the SNS as an adjunct to medical therapy, currently focused on patients with resistant hypertension and few options. Catheter-based radiofrequency (RF) ablation to effect focused tissue destruction has become commonplace in cardiac electrophysiology, interventional radiology, and tumor therapy. The prospect of trans vascular catheter-based renal denervation by radiofrequency ablation captured the imagination of interventionalists and hypertension specialists alike. Early clinical trial results portended a safe and highly efficacious therapy for resistant hypertension that seemed poised to be applied to less severe stages of the disease as well as to other clinical syndromes characterized by excessive autonomic activity, including heart failure, chronic kidney disease and obstructive 
sleep apnea. This enthusiasm has been tempered by failure of the pivotal SYMPLICITY HTN-3 study to meet its primary efficacy endpoint [3]. In this article, we will briefly explore the pre-clinical and experimental basis for renal denervation, review the current state of clinical trial results as well as limitations, and outline future directions for renal denervation.

\section{Pre-clinical and Experimental Data on Renal Nerves and SNS Activity in Hypertension}

The evidence for sympathetic nervous system activity in the development and maintenance of hypertension is compelling. In the mid-19th century, Claude Bernard identified the powerful modulating force of splanchnic nerve input on renal sodium and volume homeostasis [4], work that was extended by Bradford to demonstrate its impact on blood pressure [5]. The kidneys have a bidirectional role in this exchange. Mechanoreceptors and chemoreceptors in the renal pelvis generate signals in response to injury and other stimuli that are carried via afferent fibers to the brain and result in SNS over-activity [6]. Efferent fibers from the brain travel through the spinal cord and second sympathetic ganglia to reach the juxtaglomerular apparatus, glomerular arterioles and renal cortex with a resultant increase in sodium and water absorption, reduction in renal blood flow, and decrease in glomerular filtration rates. Campese and Kogosov demonstrated that resection of afferent renal nerves through ventral rhizotomy could prevent hypertension in rats with chronic kidney disease [7]. The importance of efferent activity was shown by O'Hagan and colleagues in deoxycorticosterone acetate (DOCA) treated miniswine with established hypertension [8]. Surgical renal denervation resulted in a normalization of pressure and natriuresis curves with blood pressure reduction. In a hyperinsulinema-induced hypertension model in rats, renal denervation prevented the development of hypertension and even normalized blood pressure once established [9]. As noted in the Introduction, the first surgical sympathectomy was performed by Adson in 1925 for treatment of malignant hypertension [10]. Around this time, others were exploring renal decapsulation and denervation for treatment of hematuria, perinephritis, and pain attributed to the kidneys [11]. The first bilateral renal denervation to treat severe hypertension was presented by Page and Heuer in 1934 [12]. The staged, bilateral procedure was done successfully. While no significant reduction in blood pressure was noted, denervation was shown to be safe and did not affect renal excretory function. A more radical interruption of sympathetic activity, thoracolumbar splanchicectomy, was implemented and demonstrated to be effective in treating malignant hypertension in about two thirds of the patients [13]. Peri-operative mortality and morbidity, however, remained high. Many patients suffered intolerable side effects including limiting postural hypotension, impotence, and bowel abnormalities. Thousands of patients underwent this treatment until the availability of oral anti-hypertensive medications in the 1950s.

Six decades later, and despite dozens of pharmacologic options acting via multiple pathways, resistant hypertension, which affects about $12 \%$ of patients with hypertension-remains a significant challenge, mirroring the increase in obesity and diabetes. With the broad emphasis in medicine over the past quarter-century on minimally-invasive alternatives to surgery, percutaneous catheter platforms have become mainstay in the treatment of cardiovascular diseases, from coronary stents to trans catheter valves to electrical mapping and ablation. The subspecialty of cardiac electrophysiology has used catheter-based radiofrequency energy delivery for more than two decades to achieve focused destruction of electrical pathways and anatomic isolation of abnormal substrate tissues. The technique uses an electrode tip connected to a generator to deliver RF energy and cause irreversible thermal injury by heating target tissue to more than $50^{\circ} \mathrm{C}$. Depth of tissue injury depends upon electrode-tissue contact, power delivery and tissue impedance. Schauerte and colleagues demonstrated the trans vascular application of RF energy for vagal nerve modification in atrial fibrillation [14]. This insight predicates the development of a catheter-based approach to SNS disruption via the renal arteries. Due to high blood flow in arteries, the endothelium and superficial medial components are sufficiently cooled during RF application but allow adequate heating to the media through which course afferent and efferent fibers. Effective implementation of trans catheter renal denervation would require complete nerve interruption by energy delivery in circumferential but longitudinally staggered fashion to avoid the possibility of subsequent renal artery stenosis. The stage was then set for clinical introduction of this promising technique.

\section{Clinical Renal Denervation Trials and Limitations}

The first-in-man SYMPLICITY HTN-1 trial was an uncontrolled, phase I study of 45 patients with resistant hypertension using the Simplicity uni-electrode system (Ardian/Medtronic) [15]. The average baseline blood pressure was 177/101 mmHG (SD 20/15 mmHG), and subjects were on an average of 4.7 anti-hypertensives with $96 \%$ taking a diuretic. Forty subjects underwent denervation with the Simplicity (Ardian/Medtronic, California) unipolar radiofrequency ablation catheter, intended to create circumferential disruption of adventitial nerve fibers through a series of longitudinal, non-overlapping lesions along each main renal artery to the ostium. An average of 4.2 and 3.7 ablations were delivered to right and left renal arteries, respectively and considered optimal, based on then available information and reinforced by the impressive $\mathrm{BP}$ reduction results. The primary outcomes were safety and change in office blood pressure at 6 months. Only a small subset had ambulatory blood pressure monitoring (12/45) or renal norepinephrine (NEPI) spillover measured by isotope dilution technique (10/45), a surrogate for renal nerve interruption. Six-month data showed good safety with no change in GFR and, beyond one intra-procedural renal artery dissection, no reno-vascular complications. Office blood pressure were lower at one month and continued to fall at six months by $-14 /-10 \mathrm{mmHG}$ and $-27 /-17 \mathrm{mmHG}$, respectively. The subset that underwent ambulatory blood pressure monitoring had a less robust average SBP reduction of $11 \mathrm{mmHG}$ compared to baseline, and another subset showed mean reduction in renal NEPI spillover of $47 \%$, reflecting only partial denervation.

The impressive results led to the SYMPLICITY HTN-2 randomized, non-blinded study of the same resistant hypertension population on an average of more than five medications [16]. 106 such patients were enrolled and followed for the primary efficacy endpoint of reduction in office blood pressure at six months. The study protocol stipulated that no changes in anti-hypertensive medications were allowed 'unless medically necessary'. As in the pilot study, investigators found an impressive reduction in office blood pressures of 32/12 $\mathrm{mmHG}$ in the $\mathrm{RDN}$ group and no change in controls. ABPM was available for fewer than half of the RDN group and showed a much smaller reduction of $11 / 7$ mmHG.

Several other early phase trials have used one or another of the six treatment platforms with CE-mark approval and available clinically in more than 80 countries. Other radiofrequency ablation systems include the single-electrode Iberis (Terumo) system and the EnligHTN 
(St. Jude), Vessix (Boston Scientific) and OneShot (Covidien) multielectrode systems, which use either an expandable basket or balloon to optimize vessel contact and achieve circumferential disruption. ReCor Medical's Paradise system uses ultrasound technology to achieve denervation. Small Phase I studies with these systems showed similar reductions in office based blood pressure measurements, although EnligHTN-1 provided systematic ABPM follow-up with a much smaller magnitude of blood pressure lowering [17]. While consistent and tantalizing, these studies raised concerns in the hypertension research community. The SYMPLICITY HTN-2 study did not show a placebo effect in the control group, which is typical in pharmacologic trials. The limited ABPM data demonstrated a much smaller effect compared to office measurements than is typically seen in studies of anti-hypertensive medications and this raised questions about the validity of the large reductions seen in office BP $[18,19]$. Finally, the absence of true control groups in these studies may have masked placebo or Hawthorne effects. Furthemore the design of many of thes single arm uncontrolled studies, allowed for "unintensional overestimation of the office BP at baseline [20].

The cardiology community learned the critical importance of a sham-controlled design in certain types of device trials. Almost twenty years ago, surgical trans myocardial laser revascularization was evaluated in uncontrolled studies of subjects having limiting angina but without anatomy suitable for traditional coronary artery bypass grafting [20,21]. An impressive reduction in Canadian Cardiovascular Society angina class was demonstrated, leading to FDA approval and clinical use. When suitable technology was developed to permit the application of this technique by catheter, this strategy was studied in the single-blinded sham-controlled DIRECT trial [22], showed no benefit in this population with angina, and proved to many the value of sham procedures in non-implant device trials.

With this in mind, the SYMPLICITY HTN-3 investigators devised and executed a randomized, single-blind, sham-controlled trial of subjects with resistant hypertension as in prior studies [3]. There was a 2:1 randomization to RDN versus sham-procedure and standard medical therapy. An assessed blinding index confirmed the effectiveness of the sham control. Blood pressure assessors were also unaware of treatment assignment. The primary endpoint was mean change in office blood pressure at 6 months, and a secondary endpoint was mean change in ABPM pressure at 6 months. 535 subjects were enrolled and taking an average of more than five anti-hypertensives at baseline. The mean change in office BP at follow-up was $-14 \pm 23$ $\mathrm{mmHg}$ in the RDN group versus $-12 \pm 26 \mathrm{mmHg}$ in the control group $(\mathrm{p}=0.26$ for between group difference). ABPM measures were also similar with mean reductions of $-6.75 \pm 15 \mathrm{mmHg}$ versus $-4.79 \pm 17$ $\mathrm{mmHg}$ in $\mathrm{RDN}$ and control groups, respectively. No differences were found by subgroup or by baseline SBP.

A number of hypotheses were offered to explain the failure of SYMPLICITY HTN-3 to meet its primary endpoint. Attenuation of the placebo effect with sham-treated controls, absence of intra-procedural feedback or endpoint, a limited role for sympathetic over activity in some hypertensives, and lack of detailed understanding of peri-renal neuroanatomical all may have contributed. The most likely explanation however is incomplete or partial denervation. A large amount odf evidence from the surgical sympathectomy literature indicate that complete renal denervation both in animal models and in humans is needed in order to achieve significant BP reduction. It may not have been realistic to attempt duplication of complete surgical denervation with a unipolar catheter given the need to blindly apply radiofrequency energy of limited depth (3-4 mm) in circumferential but longitudinally staggered fashion. Subsequent analysis of human and animal data clearly indicate that for a renal artery of 5-6 $\mathrm{mm}$ in diameter more than $6 \mathrm{RF}$ ablation lesions are needed to achieve circumferential, 4 quadrant and presumamply complete denervation. Furthermore RF ablation achieves lesioning up to 3-4 $\mathrm{mm}$ in depth. Recent data in both human and animal models indicate that sympathetic fibers are found as deep as $12 \mathrm{~mm}$ from the renal artery lumen. While there had been a paucity of published pre-clinical data using the device prior to the phase 1 and 2 trials, subsequent anatomic and experimental studies have shed some light on potential failure modes.

Tellez and colleagues described the peri-renal nerve distribution and density in miniature swine [23]. They examined renal arteries from ostia to bifurcation and radially to a depth of 10 millimeters, more extensive than previously described. About half of identified fibers were within $2.5 \mathrm{~mm}$ of the lumen-wall interface, predominating in the proximal versus mid or distal segments. Similarly, an autopsy of 20 human subjects showed greater nerve density in the proximal segments where they were distributed radially at greater depths [24], and perhaps out of the range of effective RF ablation. A study by Tzafriri, et al. shed some light on the significance of these descriptions by studying the effects of multi-electrode RF ablation in miniature swine [25]. Detailed anatomic studies indicate that sympathetic fibers are closer to the lumen distally prior to bifurcation of the renal arteries and into the branches. Experimental data from studies performing ablations distally and into the branches demonstrate great efficacy comparable to surgical denervation (reduction in tissue NEPI $>90 \%$ ).

\section{Future Directions}

For catheter based renal denervation to be effective close to complete denervation needs to be achieved. The new information that emerged from recent studies both in humans and experimental models about anatomical distribution of sympathetic fibers in the renal artery adventia, is crucial. First the distribution of fibers in certain animal models (domestic swine) is very similar to humans. Second this data indicate that more fibers are found in the proximal segment of the renal artery but some of them travel to organs other than the kidney. Third fibers are found as deep as $12 \mathrm{~mm}$ from the lumen and fourth more fibers closer to the lumen are found at the distal segment of the renal artery and into the branches.

Taking into consideration this new information it becomes inevitable that RF ablation to be successful need to adopt the technique so to reach and disrupt most of the fibers. Available ablation systems can only cause thermal injury up to $4 \mathrm{~mm}$. Thus the RF- based renal denervation needs to be performed distally and into the branches. Not surprisingly studies are underway implementing this new approach. Problems and concerns however are still present with this new approach: first there may be anatomic variations in patients and in some fibers may not be reachable even at the distal segment or into the branches. Furthermore branch ablation (in much smaller vessels) may predispose to much higher rates of renal artery stenosis. It remains to be seen whether this new approach is safe and effective in sham, controlled studies.

More promising approaches are those who can impact sympathetic fibers at any depth and in a circumferential pattern. Such a technique is the one tested by ablation solutions. This approach is using a novel device that is inserted in the renal artery through a guide catheter and allows the insertion of three 27 gauge needles through the renal artery 
wall into the adventia. Then a small amount of alcohol $(0.6 \mathrm{ml})$ can be infused which can destroy all fibers to a depth of up to $12 \mathrm{~mm}$. Alcohol is neuro-lytic and fibers are very sensitive to alcohol. This technique is promising as it can achieve complete denervation and seems safe. Early results indicate no evidence of renal artery injury, no renal artery stenosis or change in renal function and remarkable efficacy in BP reduction and medication discontinuation.

Other techniques that can achieve compete denervation are underway. So in summary future directions will focus in methods and techniques that can achieve complete renal denervation and are safe. Alcohol ablation is the first of these methods and data so far indicate that it is both safe and effective. Other neurolytic agents are under investigation and can presumably have similar results. Other sources of energy such as High Intensity Focus Ultrasound (HIFUS) may have similar applicability. It is not known if modification to RF ablation can achieve comparable fiber disruption. In any case we remain optimistic that technology and science will come together and invent safe and effective ways to achieve complete renal denervation. We believe that complete interruption of renal nerves will have dramatic effect on resistant or difficult to control hypertension and it will at the end of the day help a lot of these high risk patients.

\section{References}

1. Roger VL, Go AS, Lloyd-Jones DM, Adams RJ, Berry JD, et al. (2011) Heart disease and stroke statistics--2011 update: a report from the American Heart Association. Circulation 123: e18-e209.

2. Cutler JA, Sorlie PD, Wolz M, Thom T, Fields LE, et al. (2008) Trends in hypertension prevalence, awareness, treatment, and control rates in United States adults between 1988-1994 and 1999-2004. Hypertension 52: 818-827.

3. Bhatt DL, Kandzari DE, O'Neill WW, D'Agostino R, Flack JM, et al. (2014) A controlled trial of renal denervation for resistant hypertension. N Engl J Med 370: 1393-1401.

4. Bernard C (1859) Lessons on physiological properties and pathological alterations of the body liquids: 170-191.

5. Bradford JR (1889) The innervation of the renal blood vessels. Journal of Physiology 10: 358-363.

6. DiBona G, Esler M (2010) Translational medicine: the anti-hypertensive effect of renal denervation. American Journal of Physiology: Regulatory, Integrative and Comparative Physiology 298: R245-R253.

7. Campese VM, Kogosov E (1995) Renal afferent denervation prevents hypertension in rats with chronic renal failure. Hypertension 25: 878-882.

8. O'Hagan KP, Thomas GD, Zambraski EJ (1990) Renal denervation decreases blood pressure in DOCA- treated miniature swine with established hypertension. Am J Hypertens 3: 62-64.

9. Huang WC, Fang TC, Cheng JT (1998) Renal denervation prevents and reverses hyperinsulinemia-induced hypertension in rats. Hypertension 32: 249-254.

10. Adson A, McCraig W, Brown G (1936) Surgery in its relation to hypertension. Surgical Gynecology and Obstetrics 62: 314-31.
11. Sen S (1936) Some observations on decapsulation and denervation of the kidney. British Journal of Urology 8: 319-328.

12. Page I, Heuer G (1935) The effect of renal denervation on the level of arterial blood pressure and renal function in essential hypertension. Journal of Clinical Investigation 14: 27-30.

13. PEET MM (1947) Results of bilateral supradiaphragmatic splanchnicectomy for arterial hypertension. N Engl J Med 236: 270-277.

14. Schauerte P, Scherlag BJ, Pitha J, Scherlag MA, Reynolds D, et al. (2000) Catheter ablation of cardiac autonomic nerves for prevention of vagal atrial fibrillation. Circulation 102: 2774-2780.

15. Krum H, Schlaich M, Whitbourn R, Sobotka PA, Sadowski J, et al. (2009) Catheter-based renal sympathetic denervation for resistant hypertension: a multicentre safety and proof-of-principle cohort study. Lancet 373: 1275-1281.

16. Symplicity HTN-2 Investigators Esler MD, Krum H, Sobotka PA, Schlaich MP, et al. (2010) Renal sympathetic denervation in patients with treatment-resistant hypertension (The Symplicity HTN-2 Trial): a randomised controlled trial. Lancet 376: 1903-1909.

17. Papademetriou V, Tsioufis CP, Sinhal A, Chew DP, Meredith IT, et al. (2014) Catheter-based renal denervation for resistant hypertension: 12month results of the EnligHTN I first-in-human study using a multielectrode ablation system. Hypertension 64: 565-572.

18. Papademetriou V, Rashidi AA, Tsioufis C, Doumas M (2014) Renal nerve ablation for resistant hypertension: how did we get here, present status, and future directions. Circulation 129: 1440-1451.

19. Papademetriou V, Tsioufis C, Doumas M (2014) Renal Denervation and Symplicity HTN-3: "Dubium Sapientiae Initium" (Doubt Is the Beginning of Wisdom). Circulation Research 115: 211-214.

20. Howard JP, Shun-Shin MJ, Hartley A, Bhatt DL, Krum H, et al. (2016) Quantifying the 3 Biases That Lead to Unintentional Overestimation of the Blood Pressure-Lowering Effect of Renal Denervation. Circulation Cardiovascular Quality and Outcomes 9: 14-22.

21. Frazier OH, March RJ, Horvath KA (1999) Transmyocardial revascularization with a carbon dioxide laser in patients with end-stage coronary artery disease. N Engl J Med 341: 1021-1028.

22. Allen KB, Dowling RD, Fudge TL, Schoettle GP, Selinger SL, et al. (1999) Comparison of transmyocardial revascularization with medical therapy in patients with refractory angina. N Engl J Med 341: 1029-1036.

23. Leon M, Kornowski R, Downey W, Weisz G, Baim D, et al, (2005) A blinded, randomized, placebo-controlled trial of percutaneous laser myocardial revascularization to improve angina symptoms in patients with severe coronary artery disease. Journal of the American College of Cardiology 46: 1812-1819.

24. Tellez A, Rousselle S, Palmieri T, Rate WR, Wicks J, et al. (2013) Renal artery nerve distribution and density in the porcine model: biologic implications for the development of radiofrequency ablation therapies. Transl Res 162: 381-389.

25. Sakakura K, Ladich E, Cheng Q, Otsuka F, Yahagi K, et al. (2014) Anatomic assessment of sympathetic peri-arterial renal nerves in man. J Am Coll Cardiol 64: 635-643.

26. Tzafriri AR, Mahfoud F, Keating JH, Markham PM, Spognardi A, et al. (2014) Innervation patterns may limit response to endovascular renal denervation. J Am Coll Cardiol 64: 1079-1087. 